\title{
GC-MS Analysis of Bioactive Compounds of Seaweed Extracts Collected from Seashore of Manalmelkudi (Pudukkottai dist., Tamilnadu), responsible for Antifungal Activity
}

\author{
K. G. Anitha*, G. Arputha, G. Muthubala, R. Susithra, \\ M. Mullaivendhan and R. Anandham \\ Agricultural College and Research Institute, Tamil Nadu Agricultural University, \\ Kudumiyanmalai - 622 104, Pudukkottai, Tamil Nadu, India \\ *Corresponding author
}

\section{Keywords}

Seaweed, Antifungal acitivity, bioactive compounds, GCMS analysis

Article Info

Accepted:

20 August 2019

Available Online:

10 September 2019

\section{A B S T R A C T}

The antifungal activity of Gracilaria cervicornis, Gracilaria gracilis, Endocladia muricata is tested against Macrophomina phaseolina and Lasiodiplodia theobromae. The mean inhibion zones induced by seaweed extracts on the tested pathogens revealed that methanolic extract of $G$. cervicornis had highest zone than its acetone \& aquous extracts followed by methanoilc extract of $G$. gracilis against $M$. phaseolina whereas no inhibition by E. muricata. The methanolic extract of $G$. cervicornis recorded inhibition zone of 20.7 $\mathrm{mm}$ against $M$. phaseolina whereas in $L$. theobromae it was $17.6 \mathrm{~mm}$. But the acetone extract of G. gracilis showed $17.4 \mathrm{~mm}$ against M. phaseolina whereas it was $16.6 \mathrm{~mm}$ for G. cervicornis. The composition of bioactive compounds in the GC-MS chromatogram of these seaweed extracts were analysed and found that the phenols contributed major portion among the various fractions of the extract which contributes for the antimicrobial effect. The peaks for phenols with area \% of 38.53 and height \% of 28.58 for G. cervicornis and $32.15 \%$ and $19.56 \%$ respectively for $G$. gracilis are recorded.The chromatogram of Endocladia muricata showed no traces of phenols. G. gracilis is having appreciable amounts of fatty acids with notable height \% viz., Hexadecanoic acid (3.45\%), nHexadecanoic acid (5.10\%), Furanacetic acid (2.3\%) and in G. cervicornis Tridecanoicacid, n-Nonadecanoic acid, cyclo propane octanoic acid, Heneicosanoic acid which are also responsible for the exhibited antifungal activity.

\section{Introduction}

Marine macroalgae are characterized for their production of wide array of biocidal substances. It is recorded that more than 600 secondary metabolites have been isolated from seaweeds; among which most of them are toxic substances acting as chemical defense system for protection from their grazers. They normally produce such kinds of bioactive substances in a response to predation, competition for space and tide variations. Hence possibilities for exploring the antimicrobial compounds of seaweed origin 
for control of plant pathogens are more. These antimicrobial compounds include polysaccharides, fatty acids, phenolics, carotenoids and terpenes. In this study, an attempt has been made to characterize the antifungal activity of Gracilaria cervicornis, Gracilaria gracilis, Endocladia muricata which were collected from seashore of Manalmelkudi, Pudukkottai District, Tamilnadu, India

\section{Materials and Methods}

\section{Location of the sample}

Seaweeds were collected from seashore of Manalmelkudi, Pudukkottai district (Latitude $10.0396^{\circ} \mathrm{N}$, Longitude $79.2318^{\circ} \mathrm{E}$ ). Totally 12 types of seaweeds were collected from 5 locations.

\section{Processing of seaweed}

The collected macroalgae were washed with fresh water to remove the extraneous substances and transported to laboratory by packing in polythene bags. In the lab they were again rinsed with saline solution, shade dried and powdered in a mixer grinder. The powder was immediately used for extraction.

\section{Extraction of bioactive compounds}

Each seaweed powder was mixed with two different hydrophilic solvents (methanol, acetone) in the ratio of 1:50 (w/v). The solutions were kept in an Orbital incubator shaker (Optics Technology) at $160 \mathrm{rpm}$ for 48 hours. The extracts were filtered through Whatman No. 1 filter paper and concentrated by evaporation in vacuum. The residual extracts were stored at $0^{\circ} \mathrm{C}$.

\section{Phytochemical analysis by GC-MS}

A mass Spetrometry equipped with a data system in combination with $\mathrm{GC}$ was used for the analysis of seaweed extract. The methanolic extracts of the 3 screened seaweeds were injected $(1 \mu \mathrm{l})$ in GC-MS (Varian CP 2000) with Poropak Q column, FID detector, flow rate of $1.0 \mathrm{ml} / \mathrm{min}$. and total run time of 20 minutes. The compounds were identified from the library search result of GC-MS.

\section{Determination of antifungal activity}

The antifungal activity was determined by agar well diffusion method (Suay et al., 2000). The fungal pathogens used for this study were Macrophomina phaseolina, a root pathogen and Lasiodiplodia theobromae, a foliar pathogen. The agar plates inoculated with the test fungi were incubated for one hour before placing extracts and $80 \mu \mathrm{l}$ of seaweed extract was placed in the wells and allowed to diffuse for 2 hours. Then the plates were incubated for 72 hours. The antifungal activity was determined by measuring the diameter of inhibition zone for each well and expressed in mm.

\section{Results and Discussion}

Among the 12 seaweeds collected 3 were identified based on the morphological characters \& cross section under microscope (Marine seaweed Manual, 2018) and confirmed by Botanical Survey of India, Coimbatore as Gracilaria cervicornis, Gracilaria gracilis and Endocladia muricata.

\section{Anatagonistic activity against fungal pathogens}

In many studies it has been proved that hydrophyllic solvents provide better activity as many of the bioactive compounds are extracted by them rather than in lipophyllic solvents. Zineb et al., (2004) has reported the total inhibition of A. flavus by the ethanolic 
extract of brown marine algae, Cystoseira tamariscifolia. Cox et al., (2010) suggested for usage of methanol for brown and red seaweed extraction. Hence methanol and acetone were used for extraction in this study.

The organic solvents extract showed activity against pathogens but aqueous extract showed minimum or no activity. In general $M$. phaseolina was fond to be sensitive to the extracts of both $G$. gracilis and $G$. cervicornis than $L$. theobromae. The mean inhibion zones induced by seaweed extracts on the tested pathogens revealed that methanolic extract of $G$. cervicornis had highest zone than its acetone \& aqueous extracts followed by methanoilc extract of $G$. gracilis against $M$. phaseolina. The methanolic extract of $G$. cervicornis recorded inhibition zone of 20.7 $\mathrm{mm}$ against $M$. phaseolina whereas in $L$. theobromae it was $17.6 \mathrm{~mm}$. But the acetone extract of $G$. gracilis showed $17.4 \mathrm{~mm}$ against $M$. phaseolina whereas it was $16.6 \mathrm{~mm}$ for $G$. cervicornis (Table 1). In the present study the antifungal activity of Gracilaria is proved in first of its kind against $M$. phaseolina and $L$. theobromae.

\section{GC-MS analysis of bioactive compounds}

During the run time of 20 minutes 50 peaks were obtained. It is stated that algae produce secondary metabolites up to $7 \%$ of dry weight which show antimicrobial activity. Among these about $60 \%$ are terpenes, $20 \%$ are fatty acids along with nitrogenous compounds (Paul and Fenical, 1987; Van Alstyne and Paul, 1988). In the present study as the methanolic extracts of G. gracilis and G. cervicornis have appreciable amount of antifungal activity. The composition of bioactive compounds in the GC-MS chromatogram of these seaweed extracts were analyzed and found that they contained a mixture of compounds. The retention time, peak area $\%$, peak height $\%$ along with compound name, formula and molecular weight were fetched from the library data as given in table $2 \& 3$. Correlating the presence of certain phenolics, fatty acids and terpenes to the antifungal activity exhibited by seaweeds has been attempted.

In this study the phenols contributed major portion among the various fractions of the extract. The peaks for phenols (Fig 1a) was fetched at retention time of 17.31 with area \% of 38.53 and height $\%$ of 28.58 for $G$. cervicornis and $32.15 \%$ and $19.56 \%$ respectively for $G$. gracilis (Fig 1b). The chromatogram of Endocladia muricata showed no traces of phenols.

Both the seaweeds showed the presence of diversified fatty acids viz., hexadecanoic acid (palmitic acid), Furanacetic acid, cyclopropaneoctanoic acid, heneicosanoic acid, tridecanoic acid, nonadecanoic acid etc.

In this study, GC-MS chromatogram has shown (Fig. 2) that the methanolic extract of G. gracilis is having appreciable amounts of fatty acids with notable height $\%$ viz., Hexadecanoic acid (3.45\%), n- Hexadecanoic acid $(5.10 \%)$, Furanacetic acid $(2.3 \%)$ and in G. cervicornis Tridecanoic acid, nNonadecanoic acid, cyclo propane octanoic acid, Heneicosanoic acid. The compound ethyl isoallocholate is present in the extract of $G$. cervicornis. 3,7,11,17-Tetramethyl-2hexadecen-1-ol and 17 1,5-Dimethylhexyl)10,13-dimethyl-2,3,4 which are generally proved for antimicrobial activity.

The presence of 3,7,11,17-Tetramethyl-2hexadecen-1-ol, 17 1,5-Dimethylhexyl)-10,13dimethyl-2,3,4, Di-n-decylsulfone1, 2-Bis (trimethylsilyl) benzene compounds in chromatogram of G. cervicornis (Fig 3 a, b, c) with height $\%$ of $4.62,6.0$ and 3.8 respectively has been noted and in previous studies their antimicrobial activities have been proved 
Table.1 Inhibition zone around Macrophomina phaseolina and Lasiodiplodia theobromae fungal pathogens by solvent extracts of seaweeds

\begin{tabular}{|c|c|c|c|c|c|c|}
\hline \multirow{2}{*}{$\begin{array}{l}\text { Name of } \\
\text { Seaweed }\end{array}$} & \multicolumn{2}{|c|}{ Methanol extract } & \multicolumn{2}{|l|}{ Acetone extract } & \multicolumn{2}{|c|}{ Aqueous Extract } \\
\hline & $\begin{array}{l}\text { Macrophomina } \\
\text { phaseolina }\end{array}$ & $\begin{array}{l}\text { Lasiodiplodia } \\
\text { theobromae }\end{array}$ & $\begin{array}{l}\text { Macrophomina } \\
\text { phaseolina }\end{array}$ & $\begin{array}{l}\text { Lasiodiplodia } \\
\text { theobromae }\end{array}$ & $\begin{array}{l}\text { Macrophomina } \\
\text { phaseolina }\end{array}$ & $\begin{array}{l}\text { Lasiodiplodia } \\
\text { theobromae }\end{array}$ \\
\hline $\begin{array}{l}\text { Gracilaria } \\
\text { gracilis }\end{array}$ & $19.20 \pm 0.37$ & $17.30 \pm 0.29$ & $17.40 \pm 0.43$ & $16.20 \pm 0.39$ & $3.20 \pm 0.34$ & $2.40 \pm 0.29$ \\
\hline $\begin{array}{l}\text { Gracilaria } \\
\text { cervicornis }\end{array}$ & $20.70 \pm 0.18$ & $17.6 \pm 0.32$ & $16.6 \pm 0.41$ & $15.1 \pm 0.22$ & $4.1 \pm 0.24$ & $3.6 \pm 0.36$ \\
\hline $\begin{array}{l}\text { Endocladia } \\
\text { muricata }\end{array}$ & 0 & 0 & 0 & 0 & 0 & 0 \\
\hline
\end{tabular}

(Values $(\mathrm{mm})$ of inhibition zones are mean $\pm \mathrm{SD}$; sample $(\mathrm{n})=7$ )

Table.2 Phytochemical composition of methanolic extract of Gracilaria cervicornis by GC-MS

\begin{tabular}{rrrr} 
Peakf & R.Time & I.Time & F.Time \\
1 & 3.321 & 3.290 & 3.355 \\
2 & 3.485 & 3.445 & 3.525 \\
3 & 3.570 & 3.525 & 3.585 \\
4 & 3.708 & 3.680 & 3.750 \\
5 & 3.800 & 3.750 & 3.825 \\
6 & 5.221 & 5.200 & 5.245 \\
7 & 6.461 & 6.390 & 6.495 \\
8 & 11.610 & 11.565 & 11.675 \\
9 & 12.490 & 12.445 & 12.545 \\
10 & 12.560 & 12.545 & 12.575 \\
11 & 13.570 & 13.540 & 13.580 \\
12 & 13.623 & 13.580 & 13.665 \\
13 & 13.753 & 13.665 & 13.860 \\
14 & 13.910 & 13.860 & 13.930 \\
15 & 13.940 & 13.930 & 13.970 \\
16 & 14.230 & 14.170 & 14.275 \\
17 & 15.100 & 15.050 & 15.120 \\
18 & 15.155 & 15.120 & 15.170 \\
19 & 15.185 & 15.170 & 15.205 \\
20 & 15.245 & 15.230 & 15.265 \\
21 & 15.275 & 15.265 & 15.345 \\
22 & 15.366 & 15.345 & 15.380 \\
23 & 15.395 & 15.380 & 15.525 \\
24 & 15.552 & 15.525 & 15.585 \\
25 & 15.820 & 15.735 & 15.865 \\
26 & 15.900 & 15.865 & 15.935 \\
27 & 15.951 & 15.935 & 15.975 \\
28 & 16.124 & 15.975 & 16.220 \\
29 & 16.290 & 16.220 & 16.330 \\
30 & 16.347 & 16.330 & 16.370 \\
31 & 16.440 & 16.370 & 16.450 \\
32 & 16.467 & 16.450 & 16.480 \\
33 & 16.495 & 16.480 & 16.515 \\
34 & 16.570 & 16515 & 16.585 \\
35 & 16.62 & 16.585 & 16.655 \\
36 & 16.685 & 16.655 & 16.765 \\
37 & 16.780 & 16.765 & 16.895 \\
38 & 16.906 & 16.895 & 16.930 \\
39 & 17.319 & 17.110 & 17.425 \\
40 & 17.445 & 17.425 & 17.460 \\
41 & 17.663 & 17.645 & 17.775 \\
42 & 17.855 & 17.775 & 17.870 \\
43 & 18.106 & 17.870 & 18.150 \\
44 & 18.200 & 18.150 & 18.220 \\
45 & 18.320 & 18.300 & 18.415 \\
46 & 18.488 & 18.415 & 18.510 \\
47 & 18.570 & 18.510 & 18.595 \\
48 & 18.749 & 18.690 & 18.775 \\
49 & 19.079 & 18.920 & 19.110 \\
50 & 19.960 & 19.885 & 19.995 \\
& & & \\
& & & \\
\hline
\end{tabular}

\begin{tabular}{|c|c|c|c|}
\hline \multicolumn{4}{|c|}{ Peak Repart TIC } \\
\hline Area & Area\% & Height & $\begin{array}{r}\text { Height } \% \\
0.83\end{array}$ \\
\hline 14520 & 0.33 & 8160 & 0.83 \\
\hline 25593 & 0.59 & 7454 & 0.76 \\
\hline 22845 & 0.52 & 8188 & 0.84 \\
\hline 38346 & 0.88 & 11383 & 1.16 \\
\hline 35722 & 0.82 & 11634 & 1.19 \\
\hline 15016 & 0.34 & 8952 & 0.91 \\
\hline 18232 & 0.42 & 8452 & 0.86 \\
\hline 63818 & 1.46 & 19076 & 1.95 \\
\hline 90895 & 2.08 & 25109 & 2.56 \\
\hline 16142 & 0.37 & 10309 & 1.06 \\
\hline 52070 & 1.19 & 30234 & 3.08 \\
\hline 18362 & 4.21 & 45259 & 4.62 \\
\hline 419879 & 9.62 & 58832 & $\begin{array}{l}6.00 \\
6.00\end{array}$ \\
\hline 65543 & 1.50 & 21011 & 2.14 \\
\hline 16944 & 0.39 & 15129 & 1.54 \\
\hline 28333 & 0.65 & 11045 & 1.13 \\
\hline 20712 & 0.47 & 7603 & 0.78 \\
\hline 20627 & 0.47 & 11226 & 1.15 \\
\hline 21873 & 0.50 & 13094 & 1.34 \\
\hline 11893 & 0.27 & 8003 & 0.82 \\
\hline 29759 & 0.68 & 9680 & 0.99 \\
\hline 13368 & 0.31 & 9319 & 0.95 \\
\hline 66206 & 1.52 & 7103 & 0.72 \\
\hline 66082 & 1.51 & 25606 & 2.61 \\
\hline 57512 & 1.32 & 9120 & 0.93 \\
\hline $\begin{array}{l}32140 \\
32140\end{array}$ & 0.74 & 9996 & $\begin{array}{l}.93 \\
1.02\end{array}$ \\
\hline 28548 & 0.65 & $\begin{array}{l}99960 \\
14728\end{array}$ & $\begin{array}{l}1.02 \\
1.50\end{array}$ \\
\hline 358176 & 8.21 & 37231 & 3.80 \\
\hline 15512 & 3.56 & 28311 & 2.89 \\
\hline 49743 & 1.14 & 23841 & 2.43 \\
\hline 72423 & 1.66 & 15082 & 1.54 \\
\hline 21637 & 0.50 & 13397 & 1.37 \\
\hline 18182 & 0.42 & 10177 & 1.04 \\
\hline 33432 & 0.77 & 9470 & 0.97 \\
\hline 27413 & 0.63 & 8801 & 0.90 \\
\hline 40068 & 0.92 & 9101 & 0.93 \\
\hline 30870 & 0.71 & 7379 & 0.75 \\
\hline 10996 & 0.25 & 8344 & 0.85 \\
\hline 1680730 & 38.53 & 280087 & 28.58 \\
\hline 11057 & 0.25 & $79 \breve{936}$ & 0.81 \\
\hline 66129 & 1.52 & 12190 & 1.24 \\
\hline 24246 & 0.56 & 8437 & 0.86 \\
\hline 79500 & 1.82 & 10793 & 1.10 \\
\hline 13511 & 0.31 & 7259 & 0.74 \\
\hline 21787 & 0.50 & 7131 & 0.73 \\
\hline 41519 & 0.95 & 15023 & 1.53 \\
\hline 31091 & 0.71 & 9640 & 0.98 \\
\hline 22351 & 0.51 & 7538 & 0.77 \\
\hline 39582 & 0.91 & 7284 & 0.74 \\
\hline 3691 & 0.84 & 9997 & 1.02 \\
\hline & 0,00 & 3015 & \\
\hline
\end{tabular}

AH Name

78 3,3-Dimethoxy-2-but anon

3., 79 -Dimethoxy-2-but anone 2 -ethyl-

3.37 Ethylbenzene

3.07 Benzene, 1,3-dimethyl-

168 Decane

2.16 Decane, 2-methyl-

3.62 Heptadecane

157 Ethyld iso-allocholate

1.72 Neophytadiene

4.06 Cholesterd

17-(1,5-Dimethylhexy)-10,13-dimethyl-2,3,4,7,8,9,10,11,12,13,14,1

3.12 3,7,11,15-Tetramethyl-2-hex
1.12
5-Cholene, 3,24-dihydroxy-

(257

272 1-(+)-Ascorbic acid 2,6-diheradecanoate

1.84 n-Nonadecandic acid, pent amethyldsilyl ester
1.67 Card-20(22)-enolide, 2,3,14-trihydroxy-, (2.alpha.,3.bet a,5.alpha.)1.49 Diethyl 3-chlaro-2-hydroxypropylmalonate

1.43 Propyleneglycol monoleate

9.32 Benzylpiperazine, TMS derivative

6.31 4,7,7-Trimethylbicyclo[2.2. 1] heptan-2,3-dione, 2-oxime (syn)

3.22 Ethyl hexatriacontyl ethe

1.94 d-M annitol, 1-O-(22-hydroxydocosyl-

9.62 9-Octadecenamide, (

209 1,2-Bis(trimethylsily) benzene

4.80 6-Methyltricosane

1.62 2.beta, 4.beta,, 16.alpha--Tribromoallopregn-16-ene-3,20-dione

1.79 9,12-Tetradecadien-1-ol, (ZE), TMS derivative

3.53 Hexasloxane, $1,1,3,3,5,5,7,7,9,9,11,11$-dodecamethyl-

3.11 2H-3,9a-Methano-1-benzoxepin, octahydro-2,2,5a,9-tetramethyl-, [3

418 1.13,3

.

6.00 Phenol, 2,4-bis (1,1-dimethylethyl), phosphite (3:1)

1.39 Cyclopentene, 3,3-dimethyl-4-methylene-1,2-bisitrimethylsilyl axym

5.42 Cyclopropaneoctanoic acid, $2-[(2$-pentylcyclopropyl $)$ methyl $]-$, methy

2.87 2H-Pyran, 2-(7-heptadecynyloxy)tetralyydo-

7.37 Heneicosinoic acid, 20-oxo-, methyl ester

1.86 2-Bromolauric acid

3.06 d-Mannitol, l-decylsulfony-

2.76 Di-n-octyl phthalate

3.23 beta-n-Butylether of 11 -epi-dhydoartemisin

2.97 1,1,3,3-Tetraallyl-1,3-dislacyclobutane

. 43 Butyl 9 -octadecenoate or 9-18.

3.66 1,4-Benzenediol, 2,5-bis(1,1-dimethylethyl)- 
Table.3 Phytochemical composition of methanolic extract of Grcilaria gracilis by GC-MS

$\begin{array}{rrrr}\text { Peak } & \text { R.Time } & \text { I.Time } & \text { F.Time } \\ 1 & 3.323 & 3.245 & 3.430 \\ 2 & 3.470 & 3.430 & 3.490 \\ 3 & 3.565 & 3.490 & 3.525 \\ 4 & 3.546 & 3.525 & 3.575 \\ 5 & 3.714 & 3.680 & 3.755 \\ 6 & 3.795 & 3.755 & 3.860 \\ 7 & 4.042 & 4.020 & 4.075 \\ 8 & 9.580 & 9.530 & 9.610 \\ 9 & 11.591 & 11.545 & 11.740 \\ 10 & 11.800 & 11.740 & 11.815 \\ 11 & 11.840 & 11.815 & 11.905 \\ 12 & 11.916 & 11.905 & 11.945 \\ 13 & 12.478 & 12.420 & 12.565 \\ 14 & 13.565 & 13.535 & 13.590 \\ 15 & 13.608 & 13.590 & 13.635 \\ 16 & 13.794 & 13.770 & 13.835 \\ 17 & 13.898 & 13.845 & 13.935 \\ 18 & 14.089 & 14.040 & 14.120 \\ 19 & 14.208 & 14.120 & 14.275 \\ 20 & 14.320 & 14.275 & 14.420 \\ 21 & 14.498 & 14.420 & 14.550 \\ 22 & 14.565 & 14.550 & 14.580 \\ 23 & 14.590 & 14.580 & 14.630 \\ 24 & 15.449 & 15.400 & 15.485 \\ 25 & 15.537 & 15.485 & 15.590 \\ 26 & 15.753 & 15.715 & 15.780 \\ 27 & 15.875 & 15.860 & 15.900 \\ 28 & 15.910 & 15.900 & 15.930 \\ 29 & 15.942 & 15.930 & 15.955 \\ 30 & 15.965 & 15.955 & 15.990 \\ 31 & 16.065 & 15.990 & 16.090 \\ 32 & 16.110 & 16.090 & 16.145 \\ 33 & 16.175 & 16.145 & 16.245 \\ 34 & 16.306 & 16.245 & 16.340 \\ 35 & 16.353 & 16.340 & 16.370 \\ 36 & 16.419 & 16.400 & 16.435 \\ 37 & 16.517 & 16.495 & 16.575 \\ 38 & 16.672 & 16.660 & 16.690 \\ 39 & 16.931 & 16.920 & 16.940 \\ 40 & 17.130 & 17.110 & 17.145 \\ 41 & 17.317 & 17.145 & 17.425 \\ 42 & 17.485 & 17.425 & 17.505 \\ 43 & 17.550 & 17.505 & 17.585 \\ 44 & 18.483 & 18.420 & 18.550 \\ 45 & 18.745 & 18.725 & 18.860 \\ 46 & 18.885 & 18.860 & 19.030 \\ 47 & 19.109 & 19.030 & 19.165 \\ 48 & 19.251 & 19.165 & 19.270 \\ 49 & 19.655 & 19.630 & 19.670 \\ 50 & 19.815 & 19.760 & 19.845 \\ & & & \\ & & & \\ 5 & & \end{array}$

\begin{tabular}{rrrr}
\multicolumn{5}{c}{ Peak Repat TIC } & \\
Area & Area\% & Height & Heighto \\
50603 & 0.95 & 8028 & 0.55 \\
15741 & 0.29 & 7463 & 0.51 \\
10047 & 0.30 & 10071 & 0.69 \\
23952 & 0.45 & 10998 & 0.75 \\
47849 & 0.90 & 17150 & 1.17 \\
30833 & 0.58 & 9007 & 0.62 \\
16661 & 0.31 & 8175 & 0.56 \\
21422 & 0.40 & 9789 & 0.67 \\
955240 & 17.88 & 302074 & 20.69 \\
38385 & 0.72 & 8637 & 0.59 \\
35187 & 0.66 & 8828 & 0.60 \\
9573 & 0.18 & 8940 & 0.61 \\
214337 & 4.01 & 76657 & 5.2 \\
57061 & 1.07 & 30730 & 2.10 \\
24570 & 0.46 & 14802 & 1.01 \\
25955 & 0.56 & 12402 & 0.85 \\
39943 & 0.75 & 14896 & 1.02 \\
37539 & 0.70 & 13149 & 0.90 \\
138664 & 260 & 50437 & 3.45 \\
34469 & 0.65 & 8348 & 0.57 \\
401002 & 7.51 & 74486 & 5.10 \\
53841 & 1.01 & 33618 & 2.30 \\
47656 & 0.89 & 23067 & 1.58 \\
49174 & 0.92 & 15793 & 1.08 \\
142634 & 267 & 54865 & 3.76 \\
22206 & 0.42 & 8938 & 0.61 \\
18078 & 0.34 & 8756 & 0.60 \\
14244 & 0.27 & 9432 & 0.65 \\
14433 & 0.27 & 12511 & 0.86 \\
13776 & 0.26 & 10645 & 0.73 \\
128851 & 241 & 33803 & 2.32 \\
102433 & 1.92 & 35999 & 2.47 \\
152875 & 286 & 30491 & 2.09 \\
78821 & 1.48 & 15663 & 1.07 \\
14610 & 0.27 & 11157 & 0.76 \\
15995 & 0.30 & 10828 & 0.74 \\
37293 & 0.70 & 13963 & 0.96 \\
8455 & 0.16 & 7912 & 0.54 \\
7072 & 0.13 & 8071 & 0.55 \\
16723 & 0.31 & 9410 & 0.64 \\
171528 & 32.15 & 285630 & 19.56 \\
94261 & 1.76 & 21412 & 1.47 \\
63670 & 1.19 & 14885 & 1.02 \\
87953 & 1.65 & 25290 & 1.73 \\
41391 & 0.77 & 7345 & 0.50 \\
47999 & 0.90 & 8103 & 0.55 \\
52861 & 0.99 & 12254 & 0.84 \\
26166 & 0.49 & 8510 & 0.58 \\
12761 & 0.24 & 8598 & 0.59 \\
20280 & 0.38 & 8135 & 0.56 \\
5342973 & 100.00 & 1460151 & 100.00 \\
& & & \\
& & &
\end{tabular}

A/H Name
6.30 Pentanoic acid, 3-methyl-

2.11 3-(5,5-Dimethyl-4-methylidene-2-axo-1,3-oxazolidin-3-yl)propaneni 1.59 3-(1,3-Dihydroxyisopropyl)-1,5,8,11-tetraoxacyclotridecane 2.18 Carbamic acid, (alpha-methylbenzyl), , 1-ethyl-1-methylbutyl ester 2.79 Ethylbenzene

3.42 p-Xylene
2.04 Benzene, 1,3-dimethyl-

2.19 2-(Ethylenedioxy)ethylamine, $\mathrm{N}$-methyl-N-[4-(1-pyrrolidnyl)-2-buty: 3.16 Diethyl Phthalate

4.44 1-Oxaspiro[2.5] octan-4-one, 2,2,6-trimethyl-, cis-

1.07 , 77 -

280 Heptadecane

1.86 Neophytadiene

1.66 2-Pentadecanone, 6,10,14-trimethy-

242 1,2-Benzenedicarboxylic acid, bis(2-methylpropy) ester

268 Neophytadiene

285 Cetrimonium Bramide

275 Hexadecandic acid, methyl ester

4. 13 3,8,11-Trioxatetracyclo[4 $4.4 .1 .0(2,4) .0(7,9)]$ undecane, (1.alpha.,2.alp $5.38 \mathrm{n}$-Hexadecanoic acid

1.60 2-F uranacetic acid, tetrahydro-5-(2-hydroxypropy)-alpha.-methyl-, 207 [1,1'-Bicyclopropyl]-2-octanoic acid, 2'-hexyl-, methyl ester

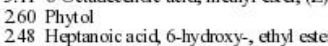

206 Silane, dimethyl $(2,2,2$-tridiloroethoxy nonydoxy-

1.51 Adamantane-1-(3,3-dichloropropyn-1-y1)

1.29 2,6-Nonadienoic acid, 7-ethyl-9-(3-ethyl-3-methylaxiranyl)-3-methyl 3.81 Octadecanamide

285 Cyclooctasiloxane, hexadecamethyl-

5.01 Diglycolic acid, pentadecyl 24,4timethyl pentyl ester

1.31 n-Hexadecanoic acid

1.31 n-Hexadecanoic acid

267 8,11,14Eicosatrienoic acid, (Z,ZZZ)-

$1.07 \mathrm{~d}-\mathrm{M}$ annitol, I-decylsal fanyl-

0.88 Sarreroside
1.77 Pregn-4-ene-20-carboxylic acid, 7,12-dihydroxy-3-oxo-, methyl este 6.01 Phenol, 2,4-bis (1,1-dimethylethyl), phosphite (3:1)

4.40 6-(2,25-Trimethyl-[1,3]dioxan-4-y1)-hepta-2,4-diencic acid methyl 4.28 Heptasloxane, hexadecamethyl-

3.48 Di-n-octyl phthalate

5.64 Di-n-decylsulfone

5.92 1,1,3,3-Tetraallyl-1,3-dislacyclobutane

4.31 trans-1,2-Diethoxycyclohexane

1.48 1,2,4-Triazol-3-amine, 5-(1,3,5-trimethyl-4-pyrazdyl)amino-

2.49 4,4-Bis(dichlorofluoromethyl)-1,2-oxathietane-2,2-dioxide 
Fig.1a GC-MS chromatogram of methanolic extract of G.cervicornis showing the presence of Phenol

«Target $\gg$

Line=:39 R.Time: 17.320 (Scan\#:2865) MassPeaks:443

RawMode:Averaged 17.315-17.325(2864-2866) BasePeal:57.10(76635)

BGMode:Calc. from Peak Group 1 - Event 1 Scan

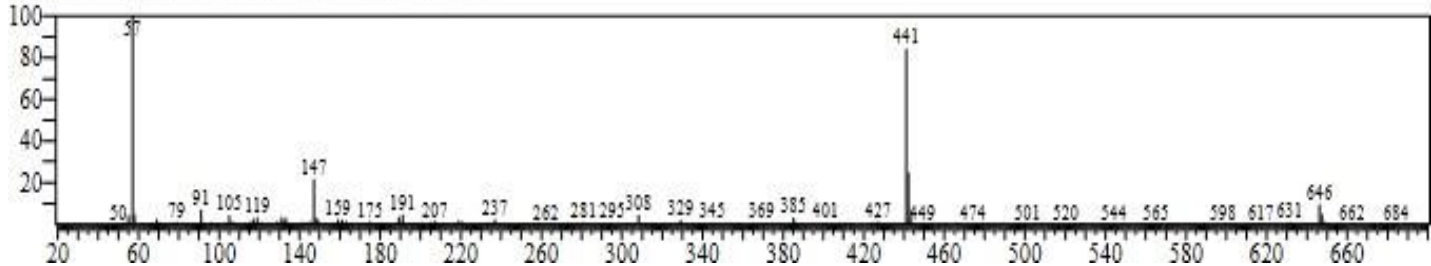

Fig 1 b. GC-MS chromatogram of methanolic extract of G. gracilis showing the presence of Phenol

$<$ Target $>>$

Line\#:41 R.Time:17.315(Scan\#:2864) MassPeaks:422

RawMode:Averaged 17.310-17.320(2863-2865) BasePeak:57.10(74382)

BG Mode:Calc. from Peak Group 1 - Event 1 Scan

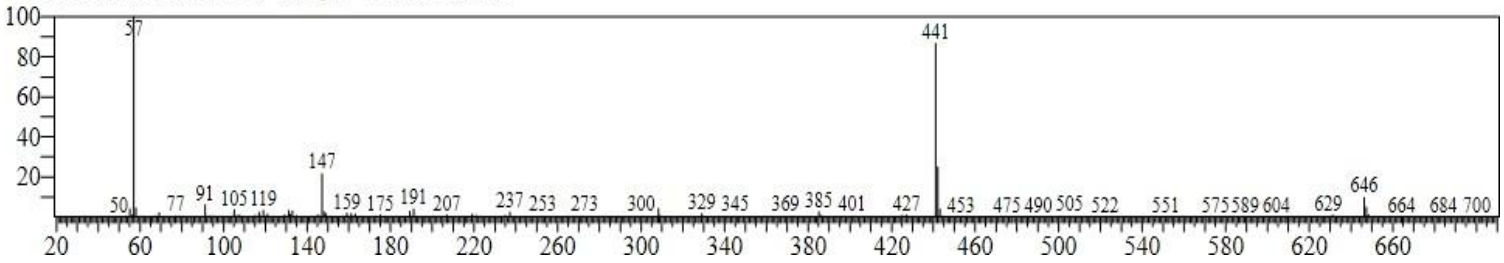

Hit\#:1 Entry:240584 Library:NIST14 lib

SI:94 Formula:C42H6303P CAS:31570-04-4 MolWeight:646 RetIndex:0

CompName:Phenol, 2,4-bis(1,1-dimethylethyl)-, phosphite (3:1) \$\$ Alkanox 240 \$\$ Hostanox PAR 24 \$\$ Lowinox 242 SS Naugard 524 \$S Tris-(2,4-di-t-bt

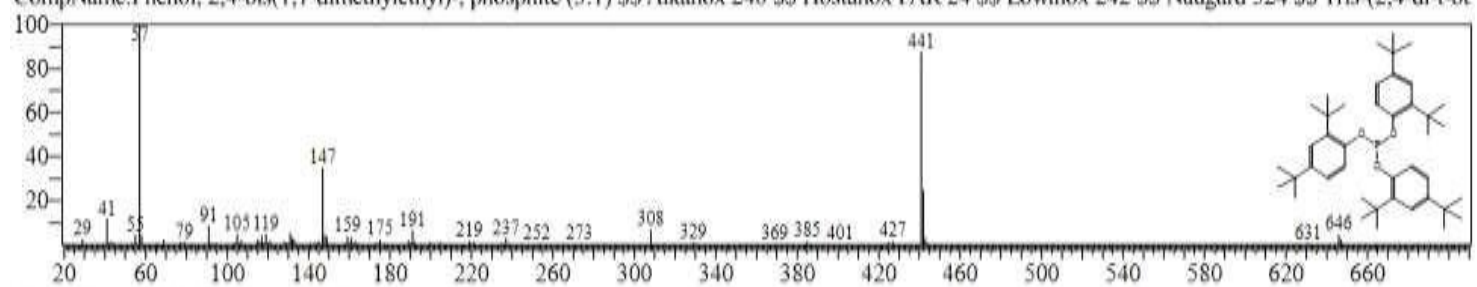


Fig 3 a. GC-MS chromatogram showing the retention time, molecular formula, molecular weight of 3,7,11,17-Tetramethyl-2-hexadecen-1-ol present in methanolic extract of G. gracilis

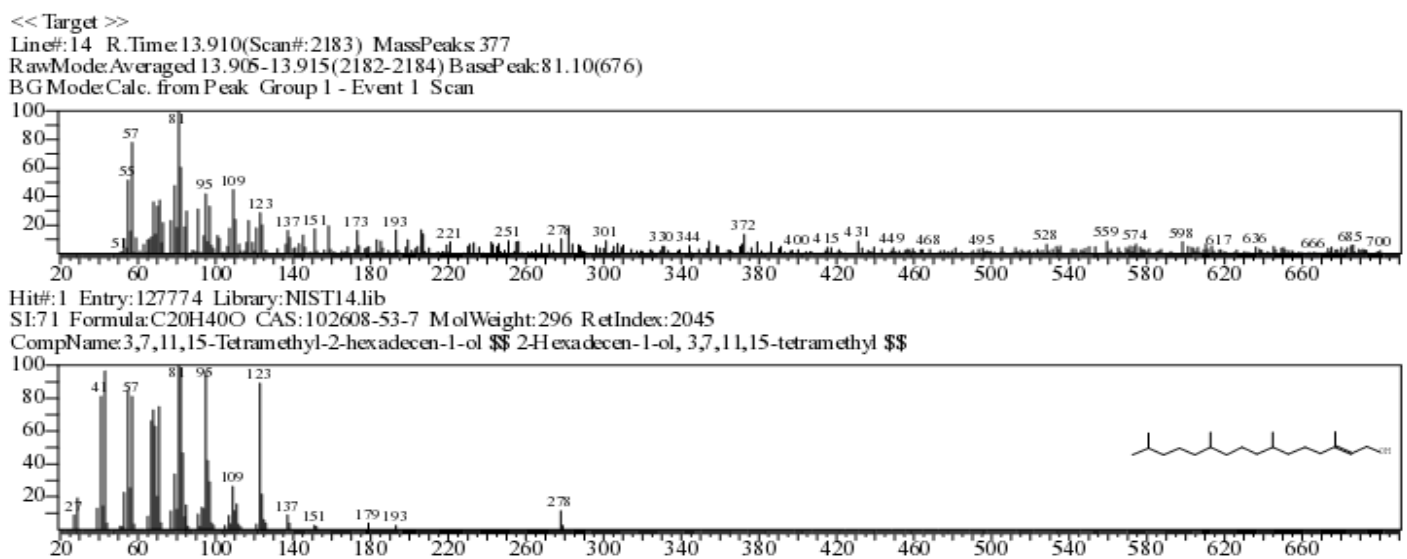

Fig 3 b. GC-MS chromatogram showing the retention time, molecular formula, molecular weight of 17 ,5-Dimethylhexyl)-10,13-dimethyl-2,3,4 present in methanolic extract of G. gracilis

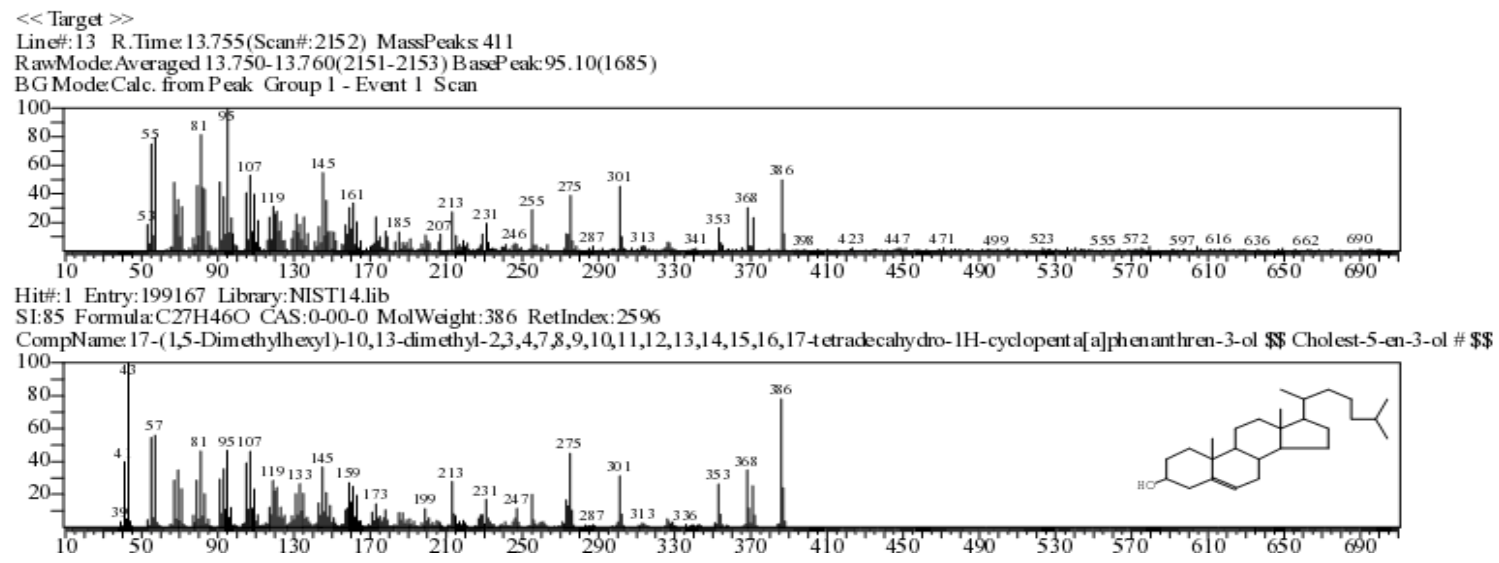

Fig 3 c. GC-MS chromatogram showing the retention time, molecular formula, molecular weight of Di-n-decylsulfone present in methanolic extract of G. gracilis

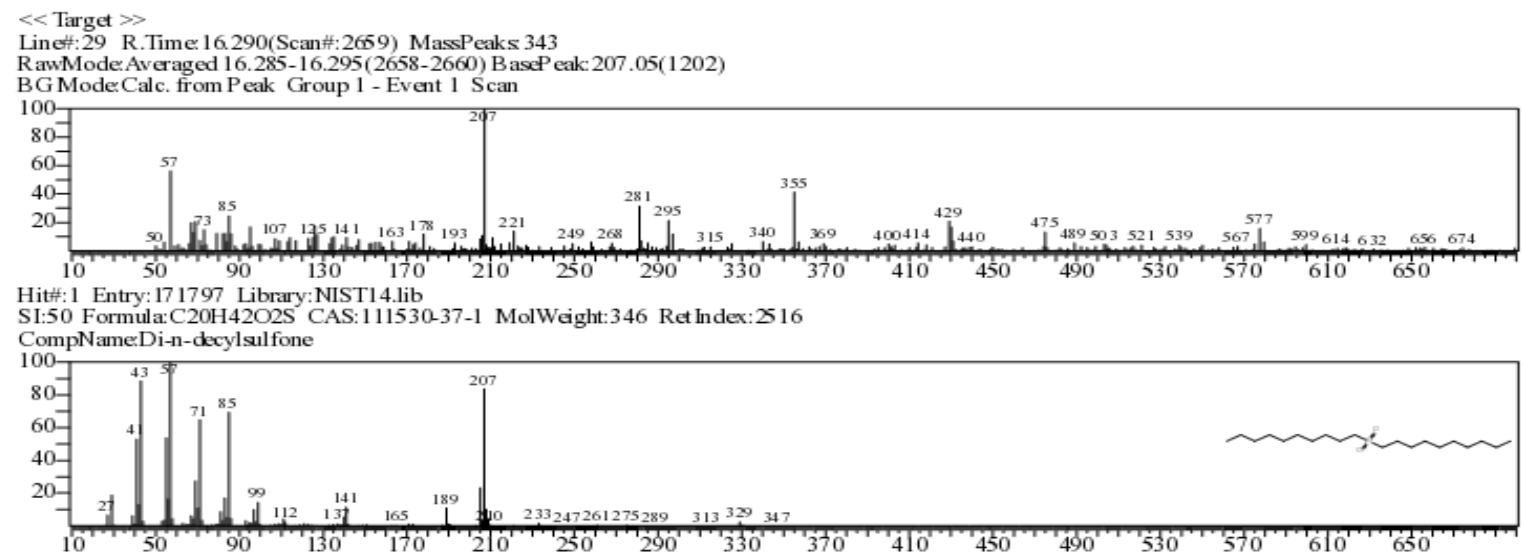


Fig.2 GC-MS chromatogram showing the retention time, molecular formula, molecular weight of Fatty acids

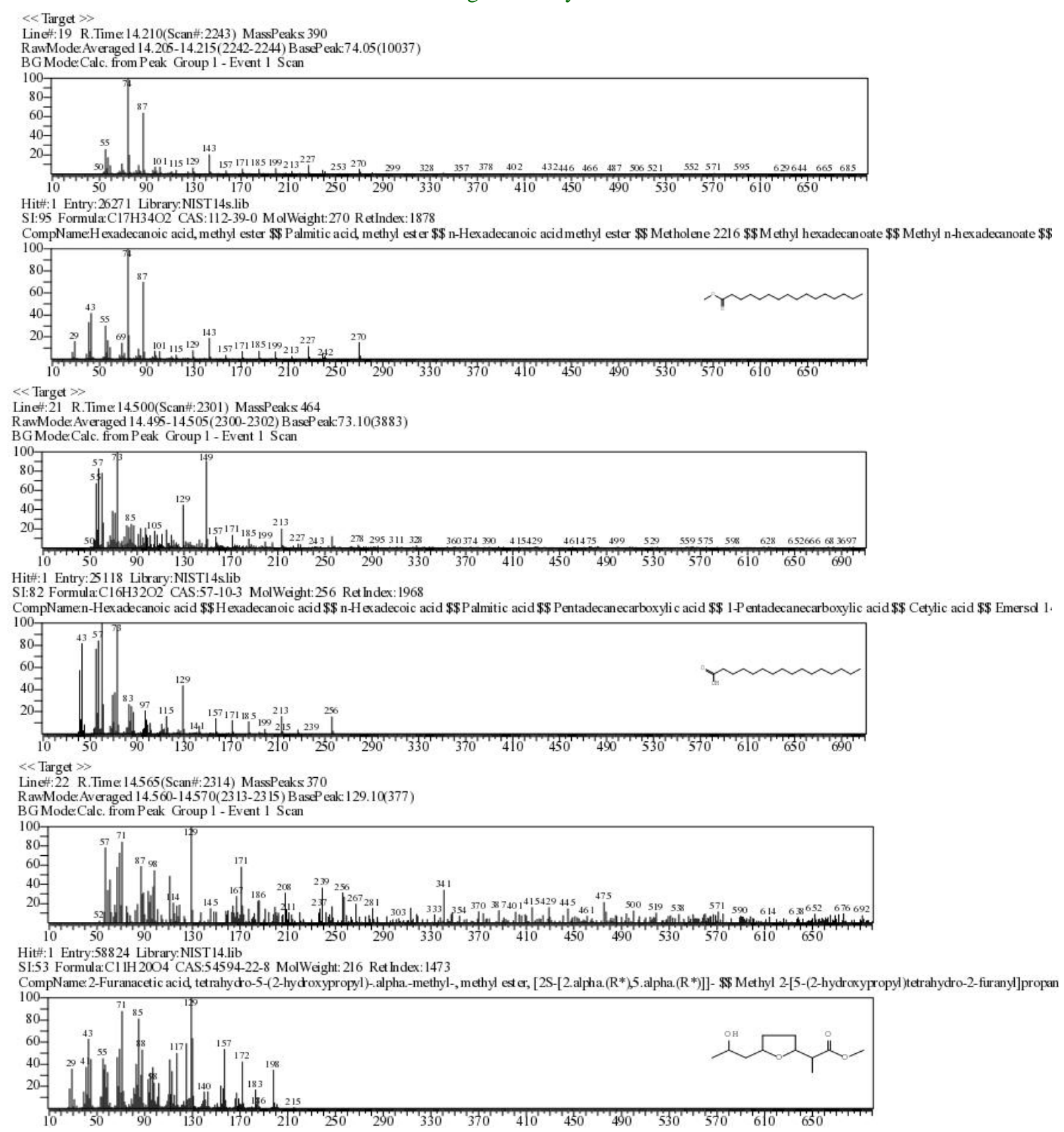

Antifungal activity against $M$. phaseolina and $L$. theobromae

Ballesteros et al., (1992) reported that most dominant plants in the mediteranean phytobentic communities such as seagrasses, Cytoseira sp, Halopteris sp, Codium sp and Mesophyllus lichenoides strongly inhibit the growth of fungi. Khaled et al., (2012) also reported that methanolic extracts of $P$. pavonica and $S$. vulgare showed antifungal effect against Candida sp strains. In one more study Saleh and Mariri (2017) proved that methanolic extract of $U$. lactuca exhibited lowest MIC value of $0.106 \mathrm{mg} \mathrm{ml}^{-1}$ against $A$. niger and Candida albicans. In line with the previous studies in this study also methanolic extract of G. cervicornis recorded inhibition 
zone of $20.7 \mathrm{~mm}$ against $M$. phaseolina and the acetone extract of the same showed 16.6 $\mathrm{mm}$. Among the two solvents used methanol seems to be effective in extraction as it could extract bioactive compounds from the seaweed efficiently. M. phaseolina is more sensitive to both seaweed extracts than $L$. theobromae.

\section{Phytochemical analysis by GC-MS}

The antimicrobial activity of phenolic compounds is attributed by changing the microbial cell permeability, leakage of macromolecules or cellular integrity loss which may lead to cell death (Abu-Ghannam and Rajauria, 2013). The presence of phenols as major portion in the extracts of both $G$. gracilis and $G$. cervicornis contributes for their antifungal activity against $M$. phaseolina and $L$. theobromae. The absence of phenols in chromatogram of Endocladia muricata strongly proves that the phenolic compounds have played main role in the antifungal activity exhibited by the other two seaweeds. The study of Ammar et al., (2017) is also in accordance with the present research finding as the phenolic acid and flavonoids in the methanolic extract of Sargassum vulgare inhibited the mycelia growth by $51 \%$ in Pythium aphanidermatum.

Aliya et al., (1995) have recorded highest amounts of tridecanoic acid and palmitic acid in Bryopisis pennata and Valoniopsis panchynema. In Laurentia brandenii, the major component of the active fraction was found to be octadecanoic acid (49.75\%) followed by hexadecanoic acid (14.24\%) and it was also observed that the higher $\%$ of octadecanoic acid contributed for the biological activity (Aseer Manilal et al., 2010). Recently, Corato et al., (2017) has demonstrated that higher fatty acid content of Laminaria digitata, Undaria pinnatifida and Porphyra umblicalis may have influence on fungal suppression as the extracts strongly reduced the incidence of brown rot of peaches and green mould on lemons. The presence of a variety of fatty acids in the extracts of both Gracilaria in this study substantiates the previous findings.

The compound ethyl isoallocholate has been shown to exhibit anti-inflammatory and antimicrobial activity (Sarada et al., 2011) and the same is also present in the extract of $G$. cervicornis used in the present study. Antibacterial property of 3,7,11,17Tetramethyl-2-hexadecen-1-ol against $A$. flavus and A.niger and 17 1,5-Dimethylhexyl)-10,13-dimethyl-2,3,4 (Santhanam et al., 2019) and biological activity including antifungal activity of Di-n-decylsulfone1, 2Bis (trimethylsilyl) benzene (Susheela Mary et $a l ., 2017)$ have been reported. The presence of these compounds in chromatogram of $G$. cervicornis (Fig $3 \mathrm{a}, \mathrm{b}, \mathrm{c}$ ) with height $\%$ of 4.62, 6.0 and 3.8 respectively substantiate for the antifungal activity exhibited by this seaweed against $M$. phaseolina and $L$. theobromae.

In the present study, the formation of inhibition zone by the methanolic extracts of G. cervicornis and G. gracilis against the root and leaf fungal pathogens viz., M. phaseolina and L. theobromae and their chromatographic cataloging shows that these two seaweeds can be effectively utilized for the extraction of antimicrobial compounds against fungal pathogens.

\section{References}

Abu-Ghannam, N. and Rajauria, G. 2013. Antimicrobial activity of compounds isolated from algae. In Functional Ingredients from Algae for Foods and Nutraceuticals. (Ed.)Domínguez, H. (ed.), Woodhead Publishing: Cambridge, UK. pp. 287-306.

Aliya, R., Shameel, M., Usmanghani, K., Sabiha, S. and Ahmed, V.U. 1995. Fatty Acid 
compositions of two siphonaceous green algae from the coast of Karachi. Pak. J. Pharm. Sci. 8(2): 47-54.

Ammar, N., Jabnoun-Khiareddine, H., Mejdoub-Trabelsibi, B., Nefzi, A., Mahjoub, M. A. and Daami-Remadi, M. 2017. Pythium leak control in potato using aqueous and organic extracts from thebrown alga Sargassum vulgare (C. Agardh, 1820). Postharvest Biol. Technol. 130: 81-93.

Aseer Manilal, S., Sujith, B., Sabarathnam, G., Kiran, S. Selvin,J., Shakir, C. and Premnath Lipton, A. 2010. Antifouling potentials of seaweeds collected from the Southwest coast of India. World J. Agri. Sci. 6(3): 243-248

Ballesteros, E., Marin, D. and Uriz, M.J. 1992. Biological activity of extracts from some mediterranean macrophytes. Bot. Mar. 35: 481-485.

Corato, U.D., Salimbeni, R., Pretis, A.D., Avella, N. and Patruno, G. 2017. Antifungal activity of crude extracts from brown and red seaweeds by a supercritical carbon dioxide technique against fruit postharvest fungal diseases. Postharvest Biology and Technolog. 131: 16-30.

Cox, S., Abu-Ghannam, N. and Shilpi, G. 2010. An assessment of the antioxidant and antimicrobial activity of six species of edible Irish seaweeds. Int.Food Res.17:205-220.

Cox, S., Abu-Ghannam, N., and Gupta, S. 2010. An assessment of the antioxidant and antimicrobial activity of six species of edible Irish seaweeds. Int. Food Res. J. 17: 205-220.

Khaled, N., Hiba, M. and Asma, C. 2012. Antioxidant and antifungal activities of $\mathrm{P}$ a din $\mathrm{a} \mathrm{p}$ a $\mathrm{v}$ o nic a and Sargassum vulgare from the Lebanese Mediterranean coast. Adv. Environ. Biol. 6: 42-48.

Paul, V.J. and Fenical, W.1987. Natural Products
Chemistry and Chemical Defense in Tropical Marine Algae of the Phylum Chlorophyta. In: Scheuder, P. J. (Ed.). Bioorganic Marine Chemistry, Spring Verlag, Berlin., pp: 1-29.

Saleh, B. and A. Al-Mariri. 2017. Phytochemical constituents of Ficus sycomorus L. and inhibitory effect of their crude extracts against bacterial pathogens. J. Nat. Prod. 10: 6-14.

Santhanam, R., Gopinath, M. and Ramesh, S. 2019. In: Santhanam, R. (Eds.) Biology and Ecology of pharmaceutical mollusks, CRC Press. Pp. 1-193

Sarada, K., Margret, R.J. and Mohan, V. 2011. GC-MS Determination of Bioactive Components of Naringi crenulata (Roxb) Nicolson. Int J Chem Tech Research. 3:15481555.

Suay, I., Arenal, F., Asensio, F.J., Basilio, A., Cabello, M.A., Diez, M.T., Garcia, J.B., Gonzalez del Val, A., Gorrochategui, J., Hernandez, P., Pelaez, F., Vicente, M.F. 2000. Screening of basidiomycetes for antimicrobial activities. Antonie van Leeuwenhoek. 78:129139.

Susheela Mary, P. and Radha, R.R. 2017. GC-MS analysis of bioactive compounds in the ethanolic extract of nest material of mead wasp, Sceliphron caementarium. International Journal of Animals and Veterinary Sciences. 11 (11): 12-17.

Van Alstyne, K.L. and Paul, V.J. 1988. The role of secondary metabolites in marine ecological interactions. In: Proc. Sixth Int. Coral Reef Conf., Townsville, Australia

Zineb, S., Mohamed, L., Mohamed, F., Khadija, F. 2004. Inhibition of growth and mycotoxins formation in Moulds by Marine Algae Cystoseira tamariscifolia. Afr. J. Biotechnol. 3(1): 71-75.

\section{How to cite this article:}

Anitha, K. G., G. Arputha, G. Muthubala, R. Susithra, M. Mullaivendhan and Anandham, R. 2019. GC-MS Analysis of Bioactive Compounds of Seaweed Extracts Collected from Seashore of Manalmelkudi (Pudukkottai dist., Tamilnadu), responsible for Antifungal Activity. Int.J.Curr.Microbiol.App.Sci. 8(09): 2042-2051. doi: https://doi.org/10.20546/ijcmas.2019.809.236 